\title{
Study of Prevalence and Antimicrobial Susceptibility Pattern of Enterococci Isolated from Clinically Relevant Samples with Special Reference to High Level Aminoglycoside Resistance (HLAR) in a Rural Tertiary Care Hospital
}

\author{
Deeksha Rana ${ }^{1}$, Suvarna Sande ${ }^{2}$ \\ ${ }^{1}$ Jawaharlal Nehru Medical College, Datta Meghe Institute of Medical Sciences (Deemed to Be University) Sawangi \\ (Meghe), Wardha, Maharashtra, India. ${ }^{2}$ Department of Microbiology, Jawaharlal Nehru Medical College, Datta \\ Meghe Institute of Medical Sciences (Deemed to Be University), Sawangi (Meghe), Wardha, Maharashtra, India.
}

\section{ABSTRACT}

\section{BACKGROUND}

Enterococci are important human pathogens that cause many infections including nosocomial infections. Some important clinical infections caused by Enterococcus species are urinary tract infections, bacterial endocarditis, genital tract infections, surgical wound infections, bacteraemia and meningitis. Around, $80-90 \%$ of infections are commonly caused by $E$. faecalis followed by E. faecium with a contribution of about $10-15 \%$ along with emergence of multi-drug resistance (MDR) including to vancomycin. Enterococci have developed both intrinsic and acquired resistance towards many antibiotics including to high level aminoglycosides. This short term project was undertaken to study the prevalence and antibiotic susceptibility (AST) pattern of Enterococcus species isolated from clinical specimen with special reference to high level aminoglycoside resistance (HLAR) in a rural tertiary care hospital.

\section{METHODS}

100 Enterococci isolated from clinically relevant samples were identified according to standard procedures and AST was carried out according to CLSI guidelines.

\section{RESULTS}

Out of 100 enterococci, 70 E. faecalis, 21 E. faecium and 09 other Enterococcus species were isolated. The results showed that majority of enterococci was isolated from $>60$ age group (37\%), from male patients (59\%), from urine samples $(59 \%)$ and from medicine department (36\%). AST showed overall high resistance to Penicillin (98\%) Ampicillin (86\%), Gentamicin (85\%), Ciprofloxacin (60\%), Vancomycin (12\%) (VRE), high level gentamicin (42\%) (HLGR) and high level streptomycin (34\%) (HLSR) and 15\% isolates showed resistance to HLGR + HLSR. Multi drug resistance was seen in $40(57.1 \%)$ E. faecalis isolates and 11 (52.3\%) E. faecium isolates. Minimum resistance was observed with Linezolid (3\%).

\section{CONCLUSIONS}

The present study showed high prevalence of antibiotic resistance in Enterococci. Hence, Enterococcus species isolated from samples should be routinely screened for HLAR, MDR and VRE so as to prevent the spread of multi drug resistant Enterococci and for proper selection of antibiotics.

\section{KEY WORDS}

Enterococci, HLGR, HLSR
Corresponding Author: Dr. Suvarna Sande, Professor, Jawaharlal Nehru Medical College, DMIMS (DU) Sawangi (Meghe), Wardha, Maharashtra, India. E-mail: suvarnasande@yahoo.co.in

DOI: $10.14260 / j e m d s / 2020 / 537$

How to Cite This Article:

Rana D, Sande S. Study of prevalence and antimicrobial susceptibility pattern of enterococci isolated from clinically relevant samples with special reference to high Level aminoglycoside resistance (HLAR) in a rural tertiary care hospital. J Evolution Med Dent Sci 2020;9(34):2472-2478, DOI: 10.14260/jemds/2020/537

Submission 24-03-2020,

Peer Review 11-07-2020,

Acceptance 17-07-2020,

Published 24-08-2020.

Copyright (C) 2020 JEMDS. This is an open access article distributed under Creative Commons Attribution License [Attribution 4.0 International (CC BY 4.0)] 


\section{BACKGROUND}

Enterococci are gram positive, ovoid, facultative anaerobic bacteria arranged in short chains or pairs which can survive harsh temperatures and grow in high salt concentrations.[1],[2] Enterococci possess several proteins secreted in the extracellular medium such as haemolysin-cytolysin, extracellular serine protease and protease gelatinase which contribute towards its virulence. ${ }^{[3]}$ Enterococci are part of normal intestinal flora of humans and have been recognized as important human pathogens causing various infections including nosocomial infections. [1],[2],[4] The Enterococci have the ability to acquire, accumulate and share extra chromosomal elements which help in encoding antibiotic resistance genes expressing virulence traits thus explaining their increasing importance as nosocomial pathogens.[5],[6] Some important clinical infections caused by Enterococcus species are urinary tract infections, bacterial endocarditis, genital tract infections, surgical wound infections, bacteraemia and meningitis.[2],[7],[8] Around, $80-90 \%$ of infections are commonly caused by E. faecalis followed by $E$. faecium with a contribution of about $10-15 \%$ along with its predominant emergence as a multi-drug resistance (MDR) towards vancomycin.[7],[9]

The Centre for Disease Control and Prevention in a survey indicated Enterococcus as the second most common causative agent of hospital acquired UTIs (13.9\%) next to E. coli.[10] Also, in an ICU setting, Enterococci can cause up to 15\% of nosocomial urinary tract infections. ${ }^{[1]}$

Traditionally, infections caused by Enterococci species were treated with combinations of cell wall active agents (e.g., penicillin) with aminoglycoside (streptomycin), yet the synergistic effects of such combination therapy failed because some strains developed high level resistance to aminoglycosides, $\beta$ lactam antibiotics and vancomycin thus forming an alliance of HLAR with multi drug resistance (MDR).[4],[9] Enterococci have developed both intrinsic and acquired resistance towards antibiotics. Intrinsic resistance is chromosomally mediated and is the characteristic feature of species whereas, acquired resistance results from either DNA mutation or acquisition of new DNA. ${ }^{[9]}$ Intrinsically, Enterococci express its tolerance against penicillinaseresistant penicillin, cephalosporins and low levels of aminoglycosides and clindamycin. Enterococci also have acquired resistance, which includes resistance to chloramphenicol, erythromycin, high level of clindamycin, tetracycline, high level of aminoglycosides, penicillin, fluroquinolones; vancomycin etc., resistance to high level aminoglycosides occurs because of the release of various aminoglycoside modifying enzymes.[11],[12] Interestingly, Enterococci are susceptible to co - trimoxazole in - vitro but not in- vivo, because of its ability to incorporate preformed folic acid which helps them to bypass the inhibition of folate synthesis produced by co - trimoxazole.[9]

Till 1970, Streptomycin was clinically regarded as the drug of choice but soon, tolerance to the drug was observed in more than half of the Enterococci strains. In 1979, resistance to high level gentamicin was first noted in a strain of E. faecalis. [1] Until recently, the drug that could be considered for the treatment of Multidrug resistant Enterococci infections was Vancomycin. However, emergence and worldwide prevalence of Vancomycin resistant Enterococci (VREs) has made treatment of serious Enterococcal infections cumbersome. Strains of VRE along with ampicillin resistance and HLAR are most difficult to treat.[13] Enterococcus posing resistance to three important antimicrobial agents (penicillin, aminoglycosides and vancomycin) is a serious challenge for both clinicians and healthcare care institutions as it leads to imprudent antibiotic use, failure of treatment, colonization pressure and spread of resistant strains of isolates.[14]

Hence, this study has been undertaken to study the prevalence and antibiotic susceptibility (AST) pattern of Enterococci species isolated from clinical specimen with special reference to high level aminoglycoside resistance (HLAR) from a rural tertiary care hospital.

\section{METHODS}

This cross - sectional study was conducted in the Department of Microbiology in a rural tertiary care hospital for 3 months [June-August 2019, ICMR-STS] after obtaining approval from Institutional Ethics Committee. Sample collection and processing duration was of 2 months (ICMR-STS). The sample size was calculated using open EPI software. Based on findings from previous study [15] about HLAR Enterococci isolates - expecting 5\% margin of error and confidence interval of $90 \%$, the sample size was calculated as 96 which was rounded off to 100 . So, 100 Enterococci isolated from various clinically significant samples [urine, blood, in dwelling catheter, pus, surgical site and body fluids etc] were included in the study.

Enterococci isolated from samples of OPD patients and samples from various environmental sources were excluded.

The details of each sample were recorded carefully in each case like details of the patients' name, age, sex, registration number, ward, diagnosis and site from which sample was collected etc. Direct microscopy which includes gram staining was carried out on appropriate samples. Samples were cultured on Nutrient Agar, Blood Agar and MacConkey Agar and incubated overnight at $37^{\circ} \mathrm{C}$.

Enterococci isolated were identified according to the guidelines of Koneman (1975) ${ }^{[16]}$ on the basis of colony characters, gram staining and biochemical test like Catalase Test, Bile Esculin Test, Arabinose and Sorbitol fermentation test, growth on $6.5 \% \mathrm{NaCl}$ agar and black coloured colonies on Potassium tellurite medium etc.

Antibiotic susceptibility profile of 100 Enterococci strains isolated from clinical samples was studied by Kirby - Bauer Disc Diffusion method as per Clinical Laboratory Standard Institute (CLSI) guidelines [17]

Colonies from pure culture of Enterococcus were taken from the plate with the help of a sterile loop and transferred to a test tube containing sterile peptone water and incubated to $37{ }^{\circ} \mathrm{C}$ for $3-4$ hours (turbidity adjusted to $0.5 \mathrm{McF}$ arland standard). Lawn culture was done on Muller - Hinton agar plate by using sterile cotton swabs dipped in the broth culture of Enterococci strains. Following commercially available antibiotic discs (HiMedia) were placed aseptically on the surface of Muller - Hinton agar plate using sterile forceps and gently pressed. The plates were incubated at 37 ${ }^{0} \mathrm{C}$ for $16-18$ hours. Next day susceptibility profile of 
Enterococci to different antibiotics was noted. The area of inhibitory zone was measured and recorded.

The results were interpreted using CLSI guidelines. [17] Multi - drug resistance (MDR) is defined as resistance to at least three drugs from a variety of antibiotic classes.

The antibiotic discs used were Penicillin (10 U), Ampicillin (10 $\mu \mathrm{g})$, Gentamicin $(10 \mu \mathrm{g})$, High Level Gentamicin $(120 \mu \mathrm{g})$, High Level Streptomycin $(300 \mu \mathrm{g})$, Vancomycin $(30 \mu \mathrm{g})$, Teicoplanin $(30 \mu \mathrm{g})$, Linezolid $(30 \mu)$, Ciprofloxacin $(5 \mu \mathrm{g})$ and Nitrofurantoin $(300 \mu \mathrm{g})$ [only in urine samples].

High level aminoglycoside resistance was detected as per CLSI.[17] guidelines using High Level Gentamicin [HLG] disc $(120 \mu \mathrm{g})$ and High-Level Streptomycin [HLS] disc $(300 \mu \mathrm{g})$. Inhibition zone of $6 \mathrm{~mm}$ was taken as resistant, $7-9 \mathrm{~mm}$ as inconclusive and $\geq 10 \mathrm{~mm}$ as susceptible.

Staphylococcus aureus ATCC 25923 and Enterococcus faecalis ATCC 29212 were used as control strains.

\section{Statistical Analysis}

The statistical analysis was carried out by relevant statistical methods like percentage method (\%).

\section{RESULTS}

\begin{tabular}{|c|c|c|c|c|c|}
\hline \multicolumn{6}{|c|}{ Age Wise Distribution } \\
\hline S.no & Age Group & E. faecalis & E. faecium & $\begin{array}{l}\text { Other sp. of } \\
\text { Enterococci }\end{array}$ & $\begin{array}{c}\text { Total } \\
\text { Enterococci in } \\
\text { percentage } \\
(\%)\end{array}$ \\
\hline 1. & $<20$ & 12 & 04 & - & $16 \%$ \\
\hline 2. & $21-30$ & 06 & - & 02 & $08 \%$ \\
\hline 3. & $31-40$ & 08 & 03 & 01 & $12 \%$ \\
\hline 4. & $41-50$ & 07 & 03 & 01 & $11 \%$ \\
\hline 5. & $51-60$ & 08 & 07 & 01 & $16 \%$ \\
\hline 6. & $>60$ & 29 & 04 & 04 & $37 \%$ \\
\hline & Total & 70 & 21 & 09 & 100 \\
\hline \multicolumn{6}{|c|}{ Gender Wise Distribution } \\
\hline & GENDER & E. faecalis & E. faecium & $\begin{array}{l}\text { Other sp. of } \\
\text { Enterococci }\end{array}$ & $\begin{array}{c}\text { Total } \\
\text { Enterococci in } \\
\text { Percentage } \\
(\%)\end{array}$ \\
\hline 1. & Male & 39 & 16 & 04 & $59 \%$ \\
\hline 2. & Female & 31 & 05 & 05 & $41 \%$ \\
\hline & Total & 70 & 21 & 09 & 100 \\
\hline \multicolumn{6}{|c|}{ Sample Wise Distribution } \\
\hline & Sample & E. faecalis & E. faecium & $\begin{array}{l}\text { Other sp. of } \\
\text { Enterococci }\end{array}$ & $\begin{array}{c}\text { Total } \\
\text { Enterococci in } \\
\text { percentage } \\
(\%)\end{array}$ \\
\hline 1. & Urine (U) & 48 & 07 & 04 & $59 \%$ \\
\hline 2. & Pus (P) & 07 & 09 & 03 & $19 \%$ \\
\hline 3. & Blood (BD) & 10 & - & 01 & $11 \%$ \\
\hline 4. & Others & 05 & 05 & 01 & $11 \%$ \\
\hline & Total & 70 & 21 & 09 & 100 \\
\hline \multicolumn{6}{|c|}{$\begin{array}{l}\text { Table 1. Age, Gender, Sample and Department Wise } \\
\text { Distribution of Enterococci }(n=100)\end{array}$} \\
\hline
\end{tabular}

Out of 100 isolates, 37 enterococci were isolated from age group of greater than 60 years followed by 16 Enterococci isolates each from age group of below 20 years and from age group between 51 - 60 years. Gender wise distribution showed 59 isolates were from male patient and 41 isolates were from female patients. Sample wise distribution showed that 59 isolates were from urine sample followed by 19 from pus sample and 11 from blood sample.
Among 59 urine isolates, $24(40.6 \%)$ of Enterococcal isolates were from catheterized patients and among $11 \mathrm{blood}$ isolates, $06(45.4 \%)$ isolates were from the paediatric age group.

Department wise distribution showed that out of 100 Enterococcal isolates, 36 Enterococci were isolated from Medicine department followed by 35 isolates from Surgery department, 13 isolates from Paediatrics department, 6 isolates from Obstetrics/Gynaecology department, 5 isolates from Neuro - Surgery department, 3 isolates from Orthopaedics department and 2 isolates from Respiratory Medicine department.

\begin{tabular}{|c|c|c|c|c|c|c|c|}
\hline \multirow{2}{*}{$\begin{array}{c}\text { Antibiotics } \\
\mathbf{n}=\mathbf{( 1 0 )}\end{array}$} & \multicolumn{2}{|c|}{$\begin{array}{c}\text { E. faecalis } \\
\mathrm{n}=(\mathbf{7 0})\end{array}$} & \multicolumn{2}{|c|}{$\begin{array}{l}\text { E. faecium } \\
n=(21)\end{array}$} & \multicolumn{2}{|c|}{$\begin{array}{l}\text { Other sp. of } \\
\text { Enterococci } \\
\mathbf{n}=(09)\end{array}$} & \multirow{2}{*}{$\begin{array}{c}\text { Total } \\
\text { Resistant } \\
\text { Enterococci } \\
\text { in } \\
\text { Percentage } \\
(\%)\end{array}$} \\
\hline & $\mathbf{S}$ & $\mathbf{R}$ & $\mathbf{S}$ & $\mathbf{R}$ & $\mathbf{S}$ & $\mathbf{R}$ & \\
\hline $\begin{array}{c}\text { Penicillin } \\
(10 \mathrm{U})\end{array}$ & $\begin{array}{c}02 \\
(2.9 \%)\end{array}$ & $\begin{array}{c}68 \\
(97.1 \%)\end{array}$ & 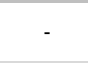 & $\begin{array}{c}21 \\
(100 \%)\end{array}$ & - & $\begin{array}{c}09 \\
(100 \%)\end{array}$ & $98 \%$ \\
\hline $\begin{array}{l}\text { Ampicillin } \\
(10 \mu \mathrm{g})\end{array}$ & $\begin{array}{c}09 \\
(12.9 \%)\end{array}$ & $\begin{array}{c}61 \\
(87.1 \%)\end{array}$ & $\begin{array}{c}04 \\
(19.1 \%)\end{array}$ & $\begin{array}{c}17 \\
(80.9 \%)\end{array}$ & $\begin{array}{c}01 \\
(11.2 \%)\end{array}$ & $\begin{array}{c}08 \\
(88.8 \%)\end{array}$ & $86 \%$ \\
\hline $\begin{array}{l}\text { Gentamicin } \\
(10 \mu \mathrm{g})\end{array}$ & $\begin{array}{c}08 \\
(11.5 \%)\end{array}$ & $\begin{array}{c}62 \\
(88.5 \%)\end{array}$ & $\begin{array}{c}05 \\
(23.9 \%)\end{array}$ & $\begin{array}{c}16 \\
(76.1 \%)\end{array}$ & $\begin{array}{c}02 \\
(22.3 \%)\end{array}$ & $\begin{array}{c}07 \\
(77.7 \%)\end{array}$ & $85 \%$ \\
\hline $\begin{array}{c}\text { High Level } \\
\text { Gentamicin } \\
(120 \mu \mathrm{g})\end{array}$ & $\begin{array}{c}40 \\
(57.2 \%)\end{array}$ & $\begin{array}{c}30 \\
(42.8 \%)\end{array}$ & $\begin{array}{c}09 \\
(42.9 \%)\end{array}$ & $\begin{array}{c}12 \\
(57.1 \%)\end{array}$ & $\begin{array}{c}09 \\
(100 \%)\end{array}$ & - & $42 \%$ \\
\hline $\begin{array}{l}\text { High Level } \\
\text { Streptomycin } \\
(300 \mu \mathrm{g})\end{array}$ & $\begin{array}{c}43 \\
(61.5 \%)\end{array}$ & $\begin{array}{c}27 \\
(38.5 \%)\end{array}$ & $\begin{array}{c}14 \\
(66.7 \%)\end{array}$ & $\begin{array}{c}07 \\
(33.3 \%)\end{array}$ & $\begin{array}{c}09 \\
(100 \%)\end{array}$ & - & $34 \%$ \\
\hline $\begin{array}{c}\text { Vancomycin } \\
(30 \mu \mathrm{g})\end{array}$ & $\begin{array}{c}62 \\
(88.6 \%)\end{array}$ & $\begin{array}{c}08 \\
(11.4 \%)\end{array}$ & $\begin{array}{c}18 \\
(85.8 \%)\end{array}$ & $\begin{array}{c}03 \\
(14.2 \%)\end{array}$ & $\begin{array}{c}08 \\
(88.9 \%)\end{array}$ & $\begin{array}{c}01 \\
(11.1 \%)\end{array}$ & $12 \%$ \\
\hline $\begin{array}{c}\text { Teicoplanin } \\
(30 \mu \mathrm{g})\end{array}$ & $\begin{array}{c}64 \\
(91.5 \%)\end{array}$ & $\begin{array}{c}06 \\
(8.5 \%)\end{array}$ & $\begin{array}{c}18 \\
(85.8 \%)\end{array}$ & $\begin{array}{c}03 \\
(14.2 \%)\end{array}$ & $\begin{array}{c}08 \\
(88.9 \%)\end{array}$ & $\begin{array}{c}01 \\
(11.1 \%)\end{array}$ & $10 \%$ \\
\hline $\begin{array}{l}\text { Linezolid } \\
(30 \mu \mathrm{g})\end{array}$ & $\begin{array}{c}68 \\
(97.2 \%)\end{array}$ & $\begin{array}{c}02 \\
(2.8 \%)\end{array}$ & $\begin{array}{c}20 \\
(95.3 \%)\end{array}$ & $\begin{array}{c}01 \\
(4.7 \%)\end{array}$ & $\begin{array}{c}09 \\
(100 \%)\end{array}$ & - & $03 \%$ \\
\hline $\begin{array}{c}\text { Ciprofloxacin } \\
(5 \mu \mathrm{g})\end{array}$ & $\begin{array}{c}27 \\
(38.6 \%)\end{array}$ & $\begin{array}{c}43 \\
(61.4 \%)\end{array}$ & $\begin{array}{c}07 \\
(33.4 \%)\end{array}$ & $\begin{array}{c}14 \\
(66.6 \%)\end{array}$ & $\begin{array}{c}06 \\
(66.7 \%)\end{array}$ & $\begin{array}{c}03 \\
(33.3 \%)\end{array}$ & \multirow{3}{*}{$\begin{array}{c}60 \% \\
\text { Total } \\
\text { Resistant } \\
\text { Enterococci } \\
\text { in percentage } \\
(\%)\end{array}$} \\
\hline \multirow{3}{*}{$\begin{array}{l}\text { Nitrofurantoin } \\
(300 \mu \mathrm{g}) \\
\text { [only in Urine } \\
\text { Sample] } \\
\mathrm{n}=(59)\end{array}$} & \multicolumn{2}{|c|}{$\begin{array}{c}\text { E. faecalis } \\
\mathrm{n}=(48)\end{array}$} & \multicolumn{2}{|c|}{$\begin{array}{c}\text { E. faecium } \\
n=(07)\end{array}$} & \multicolumn{2}{|c|}{$\begin{array}{c}\text { Other sp. of } \\
\text { Enterococci } n= \\
(04)\end{array}$} & \\
\hline & $\mathbf{S}$ & $\mathbf{R}$ & $\mathbf{S}$ & $\mathbf{R}$ & $\mathbf{S}$ & $\mathbf{R}$ & \\
\hline & $\begin{array}{c}23 \\
(48.0 \%)\end{array}$ & $\begin{array}{c}25 \\
(52.0 \%)\end{array}$ & $\begin{array}{c}05 \\
(71.5 \%)\end{array}$ & $\begin{array}{c}02 \\
(28.5 \%)\end{array}$ & $\begin{array}{c}04 \\
(100 \%)\end{array}$ & - & $46 \%$ \\
\hline \multicolumn{8}{|c|}{${ }^{*} \mathrm{~S}$ - Susceptible *R - Resistance } \\
\hline & & $\begin{array}{l}\text { e 2. Anti } \\
\text { Enteroc }\end{array}$ & $\begin{array}{l}\text { otic } \\
\text { cci I }\end{array}$ & $\begin{array}{l}\text { Resist } \\
\text { olates }\end{array}$ & $\mathrm{Po}$ & of & \\
\hline
\end{tabular}

From total 100 Enterococci species, total 98\% Enterococci isolates showed resistance to Penicillin followed by $86 \%$ isolates showed resistance to Ampicillin, $85 \%$ isolates showed resistance to Gentamicin, $60 \%$ isolates showed resistance to Ciprofloxacin, $42 \%$ isolates showed resistance to High Level Gentamicin (HLG), 34\% isolates showed resistance to High Level Streptomycin (HLS), 12\% isolates showed resistance to Vancomycin, $10 \%$ isolates showed resistance to Teicoplanin and out of total 59 Enterococci species isolated from urine sample; about $46 \%$ isolates showed resistance to Nitrofurantoin.

Out of total 100 Enterococci isolates, we found 12 (12\%) Enterococci strains resistant to Vancomycin by Kirby - Bauer disc diffusion method. We were unable to confirm the Vancomycin resistance (VRE) strains because of the non availability of E - strips and Vitek AST cards during study period. Table 2 also depicts Species wise antibiotic resistance which showed that E. faecalis was more resistant to antibiotics than $E$. faecium. 


\begin{tabular}{|c|c|c|c|c|c|}
\hline \multirow{2}{*}{$\begin{array}{l}\text { Sl. } \\
\text { No. }\end{array}$} & \multirow{2}{*}{ Antibiotics } & \multicolumn{4}{|c|}{ Samples } \\
\hline & & Urine & Pus & Blood & Others \\
\hline 1. & High Level Gentamycin & 27 & 07 & 06 & 02 \\
\hline & (HLGR) $(120 \mu \mathrm{g}) \mathrm{n}$ & $(64.2 \%)$ & $(16.6 \%)$ & $(14.2 \%)$ & $(4.7 \%)$ \\
\hline 2 . & $\begin{array}{l}\text { High Level Streptomycin } \\
(\text { HLSR) }(300 \mu \mathrm{g}) \mathrm{n}=(34)\end{array}$ & $\begin{array}{c}21 \\
(61.7 \%)\end{array}$ & $\begin{array}{c}06 \\
(17.6 \%)\end{array}$ & $\begin{array}{c}05 \\
(14.7 \%)\end{array}$ & $\begin{array}{c}02 \\
(5.8 \%)\end{array}$ \\
\hline & $\begin{array}{l}\text { 3. Sample Wise Preval } \\
\text { istance (HLAR) in Ente }\end{array}$ & $\begin{array}{l}\text { f High- } \\
\text { ci Speci }\end{array}$ & $\begin{array}{l}\text { evel Am } \\
\text { Isolate }\end{array}$ & $\begin{array}{l}\text { inoglyc } \\
s(n=7\end{array}$ & \\
\hline
\end{tabular}

\begin{tabular}{|c|c|c|c|c|}
\hline Sl. No. & & HLGR & HLSR & HLGR + HLSR \\
\hline 1. & $\begin{array}{l}\text { E. faecalis } \\
\mathrm{n}=(70)\end{array}$ & $\begin{array}{c}30 \\
(42.8 \%)\end{array}$ & $\begin{array}{c}27 \\
(38.5 \%)\end{array}$ & $\begin{array}{c}13 \\
(18.5 \%)\end{array}$ \\
\hline 2. & $\begin{array}{l}\text { E. faecium } \\
\mathrm{n}=(21)\end{array}$ & $\begin{array}{c}12 \\
(57.1 \%)\end{array}$ & $\begin{array}{c}07 \\
(33.3 \%)\end{array}$ & $\begin{array}{c}02 \\
(9.5 \%)\end{array}$ \\
\hline 3. & $\begin{array}{c}\text { Other sp. of } \\
\text { Enterococcus }=(09)\end{array}$ & - & - & - \\
\hline & Total & $42 \%$ & $34 \%$ & $15 \%$ \\
\hline
\end{tabular}

\begin{tabular}{|c|c|c|c|c|}
\hline Antibiotics & Penicillin & Ampicillin & Vancomycin & $\begin{array}{c}\text { Total Resistant } \\
\text { Enterococci in } \\
\text { Percentage (\%) } \\
\text { [Avg.] }\end{array}$ \\
\hline HLGRn = (42) & 40 (95.2\%) & \multirow{2}{*}{$\begin{array}{l}37(88.0 \%) \\
28(82.3 \%)\end{array}$} & 09 (21.4\%) & $68.2 \%$ \\
\hline HLSRn = (34) & 33 (97.0\%) & & 03 (8.8\%) & $62.7 \%$ \\
\hline \multicolumn{2}{|c|}{ 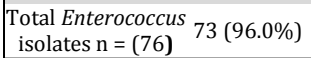 } & 65 (85.5\%) & 12 (15.7\%) & $65.7 \%$ \\
\hline \multicolumn{5}{|c|}{$\begin{array}{l}\text { Table 5. Prevalence of Enterococci Resistance to Penicillin, } \\
\text { Ampicillin and Vancomycin among HLGR and HLSR Isolates }(n=76)\end{array}$} \\
\hline
\end{tabular}

Out of 76 isolates showing HLAR, 42 isolates showed High Level Gentamicin Resistance (HLGR) from which 27 (64.2\%) were isolated from urine sample, 07 (16.6\%) from pus sample and $06(14.2 \%)$ from blood sample. 34 isolates showed High Level Streptomycin Resistance (HLSR) from which 21 (61.7\%) were isolated from urine sample, 06 (17.6\%) from pus sample and $05(14.7 \%)$ from blood sample.

Out of 70 isolates of E. faecalis, 30 (42.8\%) isolates showed High Level Gentamicin Resistance (HLGR) followed by $27(38.5 \%)$ isolates showed High Level Streptomycin Resistance (HLSR) and 13 (18.5\%) isolates showed combined resistance to High Level Gentamicin and High-Level Streptomycin (HLGR + HLSR). Out of 21 isolates of E. faecium, 12 (57.1\%) isolates showed High Level Gentamicin Resistance (HLGR) followed by 07 (33.3\%) isolates showed High Level Streptomycin Resistance (HLSR) and 02 (9.5\%) isolates showed combined resistance to High Level Gentamicin and High-Level Streptomycin (HLGR + HLSR).

Out of total 42 isolates of High-Level Gentamicin Resistance (HLGR), 95.2\% isolates showed resistance to Penicillin, 88\% isolates showed resistance to Ampicillin and $21.4 \%$ isolates showed resistance to Vancomycin. Out of 34 isolates of High-Level Streptomycin Resistance (HLSR), 97\% isolates showed resistance to Penicillin, $82.3 \%$ isolates showed resistance to Ampicillin and $8.8 \%$ isolates showed resistance to Vancomycin.

\section{Multi - Drug Resistance (MDR) Profile among Enterococci Isolates}

Out of 70 E. faecalis strains, 40 (57.1\%) isolates showed multidrug resistance i.e. resistance to at least three drugs from a variety of antibiotic classes. Out of 21 E. faecium strains, 11 (52.3\%) isolates showed MDR. So, total 51 (51\%) Enterococcal isolates showed Multidrug Resistance. Among the samples, the MDR strains were mostly isolated from urine samples (12\%) and among the wards, MDR strains were mostly isolated from Paediatric wards (53.8\%) and Medicine wards (33.3\%).

\section{DISCUSSION}

Enterococcus is a species of substantial medical importance and is a prototypical multidrug resistance pathogen and is recognized for its ubiquity and is notorious for being intrinsically advanced antibiotic resistance. Although a wide variety of antimicrobial agents with antibacterial activities are available, life threatening conditions caused by Enterococci continue to be prevalent. It is the reason for high mortality rate as it has arisen as a vital pathogen.

In present study, total 100 Enterococci species were isolated from various clinically significant samples, from the hospitalized patients and their antimicrobial susceptibility pattern was determined. Most of the isolates belonged to elderly age group of $>60$ years $(37 \%)$ followed by younger age group of $<20$ years (16\%) and older age group of $51-60$ years $(16 \%)$. The least number of isolates were found in the age group of 21 - 30 years (8\%). (TABLE 1) Decreased immunity, prolonged hospitalization and other associated co - morbidities can be relatable cause in the age group $>60$ years.

In present study, prevalence of Enterococci infection was found to be more in males (59\%). (Table 1) This result was in accordance with the study of Abdulhakim Abamecha et al [18] which reported Enterococcal infection in 50.7\% males and Varun Goel et al [19] also reported maximum cases in males (53.9\%).

Present study showed that maximum Enterococci were isolated from urine sample (59\%) followed by pus sample (19\%) and blood sample (11\%). (Table 1) Study by Deepika Atray et al ${ }^{[20]}$ reported maximum Enterococci isolates from urine (50\%) followed by pus (20\%) and blood (10\%).

In present study, out of total 59 Enterococcal isolates from urine sample, maximum number of isolations was of $E$. faecalis $48(81.3 \%)$ followed by $07(11.8 \%)$ isolates of $E$. faecium. However, out of total 19 enterococcal isolates from pus samples, maximum isolation was of E. faecium 09(47.3\%) followed by 07 (36.8\%) isolates of E. faecalis. Out of total 11 enterococcal isolates from blood samples, maximum isolation was of E. faecalis 10 (90.9\%) and no E. faecium isolates were reported. Other species of Enterococcus reported were 04 (6.7\%) isolates in urine sample, 03 (15.7\%) isolates in pus sample and $01(9 \%)$ isolates in blood sample. (Table 1)

Among 59 urine isolates, 24 (40.6\%) Enterococcal isolates were from catheterized patients and among $11 \mathrm{blood}$ isolates, $06(45.4 \%)$ isolates were from the paediatric age group which is in accordance with the study by S. Sreeja et $\mathrm{al}^{[21]}$ who reported $38 \%$ of Enterococcal isolates from catheterized patients and $58 \%$ isolates from paediatric age group.

In present study, $36 \%$ of Enterococci isolates were from medicine department followed by surgery department (35\%) and paediatrics department (13\%) which was in accordance with the study done by A. Tripathi et al ${ }^{[22]}$ who reported Enterococci isolation from medicine department up to 
$52.33 \%$ and $39.26 \%$ of Enterococcal isolation from surgery department.

In present study, overall resistance pattern showed that $98 \%$ of isolates were resistant to Penicillin followed by $86 \%$ to Ampicillin, $85 \%$ to Gentamicin, $60 \%$ to Ciprofloxacin, $42 \%$ to High Level Gentamicin, 34\% to High Level Streptomycin, $12 \%$ to Vancomycin, $10 \%$ to Teicoplanin and with lowest resistance of $3 \%$ to Linezolid. $46 \%$ of urine isolates show resistance to Nitrofurantoin. (Table 2)

\begin{tabular}{|c|c|c|c|c|c|c|c|c|c|c|}
\hline $\begin{array}{l}\text { Various } \\
\text { Study }\end{array}$ & $\mathbf{P}$ & AMP & GEN & HLG & HLS & V & TE & LZ & CIP & NIT \\
\hline $\begin{array}{l}\text { Kalpana Devi } \\
\text { Venkatesan } \\
\text { et al[ }{ }^{[23]}\end{array}$ & $\begin{array}{c}49.7 \\
\%\end{array}$ & $\begin{array}{l}21.7 \\
\%\end{array}$ & - & $\begin{array}{c}37.2 \\
\%\end{array}$ & - & $\begin{array}{l}5.7 \\
\%\end{array}$ & - & $\begin{array}{l}0 \\
\%\end{array}$ & $\begin{array}{l}56 \\
\%\end{array}$ & $\begin{array}{c}13.1 \\
\%\end{array}$ \\
\hline $\begin{array}{c}\text { Kheya } \\
\begin{array}{c}\text { Mukherjee } \\
\text { al[24] }\end{array} \text { et }\end{array}$ & - & $\begin{array}{l}70 \\
\%\end{array}$ & $\begin{array}{c}91.4 \\
\%\end{array}$ & - & - & $\begin{array}{c}3.8 \\
\%\end{array}$ & - & $\begin{array}{c}0 \\
\%\end{array}$ & $\begin{array}{l}92 \\
\%\end{array}$ & - \\
\hline $\begin{array}{c}\text { Mitali R } \\
\text { Maradia } \\
\text { et al[25] }\end{array}$ & $\begin{array}{c}97.44 \\
\%\end{array}$ & $\begin{array}{c}91.6 \\
7 \%\end{array}$ & - & $\begin{array}{c}29.49 \\
\%\end{array}$ & - & $\begin{array}{c}3.2 \\
\%\end{array}$ & $\begin{array}{c}1.92 \\
\%\end{array}$ & $\begin{array}{c}0 \\
\%\end{array}$ & $\begin{array}{c}76.28 \\
\%\end{array}$ & - \\
\hline $\begin{array}{l}\text { Seema Mittal } \\
\text { et al[26] }\end{array}$ & $\begin{array}{c}66.67 \\
\%\end{array}$ & - & - & $\begin{array}{l}29 \\
\%\end{array}$ & $\begin{array}{l}35 \\
\%\end{array}$ & $\begin{array}{c}5 \\
\%\end{array}$ & - & $\begin{array}{c}1 \\
\%\end{array}$ & $\begin{array}{l}25 \\
\%\end{array}$ & $\begin{array}{c}24.3 \\
\%\end{array}$ \\
\hline $\begin{array}{c}\text { VA Rahangdale } \\
\text { et al[27] }\end{array}$ & $\begin{array}{c}89.43 \\
\%\end{array}$ & $\begin{array}{c}43.9 \\
\%\end{array}$ & - & $\begin{array}{c}49.59 \\
\%\end{array}$ & $\begin{array}{c}47.96 \\
\%\end{array}$ & $\begin{array}{c}11.38 \\
\%\end{array}$ & - & $0 \%$ & - & $\begin{array}{c}22.76 \\
\%\end{array}$ \\
\hline $\begin{array}{c}\text { Jahnabi Barman } \\
\text { et al[28] }\end{array}$ & $\begin{array}{c}100 \\
\%\end{array}$ & $\begin{array}{c}93.6 \\
\%\end{array}$ & - & - & 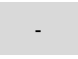 & $\begin{array}{c}1 \\
\%\end{array}$ & $\begin{array}{c}1.1 \\
\%\end{array}$ & $\begin{array}{c}0 \\
\%\end{array}$ & $\begin{array}{c}69.9 \\
\%\end{array}$ & $\begin{array}{c}15.9 \\
\%\end{array}$ \\
\hline $\begin{array}{l}\text { Aasish Karna } \\
\text { et al[29] }\end{array}$ & $\begin{array}{c}40.7 \\
\%\end{array}$ & $\begin{array}{c}39.5 \\
\%\end{array}$ & - & $\begin{array}{c}18.7 \\
\%\end{array}$ & - & $\begin{array}{c}20.9 \\
\%\end{array}$ & $\begin{array}{l}4.4 \\
\%\end{array}$ & $2.2 \%$ & $\begin{array}{c}61.5 \\
\%\end{array}$ & $\begin{array}{c}17.9 \\
\%\end{array}$ \\
\hline Present Study & $\begin{array}{l}98 \\
\%\end{array}$ & $\begin{array}{l}86 \\
\%\end{array}$ & $\begin{array}{l}85 \\
\%\end{array}$ & $\begin{array}{l}42 \\
\%\end{array}$ & $\begin{array}{l}34 \\
\%\end{array}$ & $\begin{array}{l}12 \\
\%\end{array}$ & $\begin{array}{l}10 \\
\%\end{array}$ & $\begin{array}{c}3 \\
\%\end{array}$ & $\begin{array}{l}60 \\
\%\end{array}$ & $\begin{array}{l}46 \\
\%\end{array}$ \\
\hline $\begin{array}{l}E \\
E n\end{array}$ & npa & 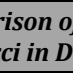 & 8 & 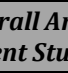 & tibic & 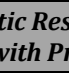 & & сеP & tte & \\
\hline
\end{tabular}

Present study reports $42 \%$ of isolates resistant to HLG and $34 \%$ isolates resistant to HLS (Table 2) which was in accordance with the study by Smeeta Huidrom et al [30] who reported that $33.3 \%$ and $36.2 \%$ of enterococci were resistant to HLGR and HLSR respectively.

In present study, $12 \%$ of isolates showed resistance to Vancomycin and $10 \%$ of isolates showed resistance to Teicoplanin. (Table 2) Study by VA Rahangdale et al [27] reports $11.3 \%$ of Vancomycin resistance and study by Maninder Kaur Jagdev et al [31] reports 5.7\% of Teicoplanin resistance which is in accordance with the present study.

Present study reports 3\% of Enterococcal isolates resistant to Linezolid (Table 2) which is accordance with the study by Aasish Karna et al[29] who reported Linezolid resistance in $4.9 \%$ isolates.

In the present study, HLGR was shown by total 42 Enterococcal isolates in which 27 (64.2\%) were isolated from urine samples followed by 07 (16.6\%) from pus samples and $06(14.2 \%)$ from blood samples (Table 3) which is higher than the study by Seema Mittal et al [26] which reported HLGR more common in urine samples $(17 \%)$ followed by pus samples (15.7\%).

In present study, $42.8 \%$ E. faecalis isolates showed HLGR resistance (Table 4) which was in accordance with the study by S. Sreeja et al [21] which showed HLGR resistance by $44.3 \%$ isolates of E. faecalis. However, the study by Maninder Kaur Jagdev et al [31] showed HLGR resistance by $82.5 \%$ E. faecalis isolates. In present study, $38.5 \%$ E. faecalis isolates showed HLSR resistance which was in accordance with the study by Smeeta Huidrom et al [30] which showed HLSR resistance by $33.3 \%$ isolates of $E$. faecalis.

In Present study, $57.1 \%$ E. faecium isolates showed HLGR resistance (Table 4) which was in accordance with the study by S. Sreeja et al ${ }^{[21]}$ which showed HLGR resistance by $54.1 \%$ isolates of E. faecium. However, the study by Maninder Kaur Jagdev et al [31] showed HLGR resistance by $92.5 \%$ E. faecium isolates. In present study, 33.3\% E. faecium isolates showed HLSR resistance which was in accordance with the study by Smeeta Huidrom et al [30] which showed HLSR resistance by $37.3 \%$ isolates of E. faecium.

In present study, other Enterococci species showed no HLGR and HLSR resistance (Table 4) but in the study by Maninder Kaur Jagdev et al [31] HLGR resistance was detected in $66.6 \%$ isolates of other Enterococci species and in the study by Varun Goel et al [19] HLSR resistance was detected in $50 \%$ isolates of other Enterococci species.

In the present study, out of 100 Enterococcal isolates, $42 \%$ isolates showed HLGR, $34 \%$ isolates showed HLSR and $15 \%$ isolates showed HLGR + HLSR. The combined resistance to both the aminoglycosides was showed by $18.5 \%$ E. faecalis isolates and $9.5 \%$ E. faecium isolates (Table 4 ) while study by DK Mendiratta et al [32] who reported combined resistance to both the aminoglycosides by $59.1 \%$ isolates of E. faecium and $7.8 \%$ isolates of $E$. faecalis. HLGR and HLSR in the present study is $42 \%$ and $34 \%$ respectively, which is alarmingly high percentage of resistance and is worthy of note.

Prevalence of HLAR in cell wall active agents like Penicillin, Ampicillin and Vancomycin was also studied. (Table 5) In the present study, out of 42 HLGR Enterococci isolates, resistance to Penicillin, Ampicillin and Vancomycin was shown by $95.2 \%, 88 \%$ and $21.4 \%$ of HLGR Enterococci isolates respectively. Also, out of 34\% HLSR Enterococci isolates, $97 \%$ of isolates showed resistance to Penicillin, $82.3 \%$ of isolates showed resistance to Ampicillin and only $8.8 \%$ of isolates showed resistance to Vancomycin. Hence, resistance to the cell wall active agents was quite high in both the species and it was higher to Penicillin than Ampicillin.

Resistant to Penicillin and Ampicillin which is usually intrinsic is due to low affinity of the penicillin - binding proteins (PBP) and it results in loss of synergistic effect between $\beta$ lactams and aminoglycosides leading to treatment failures.

The bacterial strains that show resistance to three or more categories of antibiotics are defined as multidrug resistant (MDR) strains. Multidrug resistant (MDR) Enterococci is a growing problem. Altered drug sites, bacterial efflux pumps, enzyme production or inhibition, loss of membrane protein etc. are different mechanisms mediated by MDR Enterococci.

In present study, $40(57.1 \%)$ strains of E. faecalis and 11 (52.3\%) strains of E. faecium showed MDR which was in accordance with the study by DK Mendiratta et al [32] who reported multi drug resistance of $E$. faecalis strains higher than the strains of E. faecium. However, the study by Aasish Karna et al [29] reported prevalence of MDR highest in $E$. faecium (62\%) than in E. faecalis strains.

\section{Limitations}

Present study was of short duration ( 3 months ICMR STS) and sample size was 100 isolates, studies involving large sample size and more duration to know about prevalence and changing pattern of antibiogram of Enterococcus will be more 
appropriate. Therefore, further studies should be multicentric and include a large sample size.

Vancomycin resistance (VRE) in this study was detected by Kirby - Bauer Disc Diffusion method only. We were unable to confirm the Vancomycin resistance (VRE) strains because of the non - availability of E-strips and Vitek AST cards during study period. Detection of VRE by E-strips and Vitek AST cards will be more appropriate as these methods are considered as Gold Standard methods.

\section{CONCLUSIONS}

The present study showed high prevalence of antibiotic resistance in Enterococci. Hence, Enterococcus species isolated from samples should be routinely screened by all Clinical Microbiology Laboratories for HLAR, MDR and VRE so as to prevent the spread of multiple antibiotic resistant enterococci. A regular surveillance of drug resistance can be considered for selection of antibiotics, changes in the antibiotic susceptibility patterns, and usage of antibiotics like vancomycin and linezolid in the treatment of life-threatening infections caused by Enterococci.

Authors are thankful to Indian Council of Medical Research (ICMR) for selecting this research project under ICMR - STS 2019-20. Authors are thankful to DMIMS (DU) for the help while conducting the study.

\section{REFERENCES}

[1] Murray BE. The life and times of the Enterococcus. Clin Microbial Rev 1990;3(1):46-65.

[2] Clinical updates in infectious diseases. April 1998.

[3] Arias CA, Murray BE. The rise of the Enterococcus: beyond vancomycin resistance. Nat Rev Microbiol 2012; 10(4):266-78.

[4] Murray BE. Vancomycin-resistant enterococcal infections. N Eng J Med 2000; 342(10):710-21.

[5] Sharifi Y, Hasani A, Ghotaslou R, et al. Virulence and antimicrobial resistance in enterococci isolated from urinary tract infections. Advanced Pharmaceutical Bulletin 2013; 3(1):197-201.

[6] Klibi N, Gharbi S, Masmoudi A, et al. Antibiotic resistance and mechanisms implicated in clinical Enteroccoci in a Tunisian hospital. Journal of Chemotherapy 2006; 18(1):20-6.

[7] Koneman EW, Allen SD, Janda WM, et al. The gram positive cocci Part II: Streptococci, Enterococci and the "Streptococcus -like" bacteria. Chapter - 12. Color Atlas and Textbook of Diagnostic Microbiology. $5^{\text {th }}$ edn. New York: JB Lipincott 1997: p. 577-649.

[8] Forbes BA, Sahm DF, Weissfeld AS. Laboratory methods for detection of antibacterial resistance. Chapter - 18. Bailey and Scott's Diagnostic Microbiology. 10 $10^{\text {th }}$ edn. London: Mosby 1998: p. 250-72.
[9] Morris JG Jr, Shay DK, Hebden JN, et al. Enterococci resistant to multiple antimicrobial agents, including vancomycin. Establishment of endemicity in a university medical center. Ann Intern Med 1995; 123(4):250-9.

[10] Oberoi L, Aggarwal A. Multidrug resistant enterococci in a rural tertiary care hospital - a cause of concern. JK Science 2010; 12:157-8.

[11] Marothi YA, Agnihotri H, Dubey D. Enterococcal resistance - an overview. Indian J Med Microbiol 2005; 23(4):214-9.

[12] Shah L, Mulla S, Patel KG, et al. Prevalence of enterococci with higher resistance level in a tertiary care hospital: a matter of concern. Natl J Med Res 2012; 2(1):25-7.

[13] Prakash VP, Rao SR, Parija SC. Emergence of unusual species of enterococci causing infections, South India. BMC Infect Dis 2005; 5:14.

[14] Karmarkar MG, Gershom ES, Mehta PR. Enterococcal infections with special reference to phenotypic characterization and drug resistance. Indian J Med Res 2004 ;(Suppl 119):22-5.

[15] Adhikari L. High-level aminoglycoside resistance and reduced susceptibility to vancomycin in nosocomial Enterococci. J Glob Infect Dis 2010; 2(3):231-5.

[16] Koneman EW, Allen SD, Janda WM, et al. Color atlas and textbook of Diagnostic Microbiology. Chapter - 15. Antimicrobial susceptibility testing. $5^{\text {th }}$ edn. Philadelphia: Lippicott-Raven Publishers 1997: p. 785844.

[17] Clinical and Laboratory Standard Institute. Performance standards for antimicrobial susceptibility testing: Twenty-second informational supplement. Wayne, PA: CLSI document M100, 2017; 32(3):S22.

[18] Abamecha A, Wondafrash B, Abdissa A. Antimicrobial resistance profile of Enterococcus species isolated from intestinal tracts of hospitalized patients in Jimma, Ethiopia. BMC Res Notes 2015; 8:213.

[19] Varun G, Dinesh K, Rajendra K, et al. Community acquired enterococcal urinary tract infections and antibiotic resistance profile in North India. J Lab Physicians 2016; 8(1):50-4.

[20] Atray D, Sharma A, Atray M. Prevalence of enterococci and its antibiotic resistance in various clinical samples at tertiary care hospital in Southern Rajasthan, India. Int J Res Med Sci 2016; 4(8):3413-6.

[21] Sreeja S, Babu SPR, Prathab AG. The prevalence and the characterization of the Enterococcus species from various clinical samples in a tertiary care hospital. Journal of Clinical and Diagnostic Research 2012; 6(9):1486-8.

[22] Tripathi A, Shukla SK, Singh A, et al. Prevalence, outcome and risk factor associated with vancomycin-resistant Enterococcus faecalis and Enterococcus faecium at a tertiary care hospital in Northern India. Ind J Med Microbiol 2016; 34(1):38-45.

[23] Venkatesan KD, Chander S, Ananthi B, et al. Antibiotic resistance pattern of Enterococcal isolates from patients with urinary tract infection. International Journal of Biomedical Research 2017; 8(6):357-60. 
[24] Mukherjee K, Bhattacharjee D, Chakraborti G, et al. Prevalence and antibiotic susceptibility pattern of Enterococcus species from various clinical samples in a tertiary acre hospital in Kolkata. International Journal of Contemporary Medical Research 2016; 3(6):1565-7.

[25] Maradia MR, Mehta K, Prajapati K, et al. Prevalence of multidrug-resistant Enterococcus species isolated from urine samples in a tertiary care hospital, Western India. Int J Med Sci Public Health 2017; 6(4):715-9.

[26] Mittal S, Singla P, Deep A, et al. Vancomycin and high level aminoglycoside resistance in Enterococcus spp. in a tertiary health care centre: a therapeutic concern. J Pathogens 2016; 2016:8262561.

[27] Rahangdale VA, Agarwal G, Jalgaonkar SV. Study of antimicrobial resistance in enterococci. Indian J Med Microbiol 2008; 26(3):285-7.

[28] Barman J, Nath R, Saikia L. Drug resistance in Enterococcus species in a tertiary level hospital in Assam, India. Indian J Med Res 2016; 143(1):107-10.
[29] Karna A, Baral R, Khanal B. Characterization of clinical isolates of Enterococci with special reference to glycopeptide susceptibility at a tertiary care center of eastern Nepal. Hindawi International Journal of Microbiology 2019; 2019:7936156.

[30] Huidrom S, Narayanswamy G, Dadlani R. Detection of high level aminoglycoside resistant pattern of Enterococci isolated from urine samples at a tertiary care hospital in Bengaluru. Ann Trop Med Public Health 2016; 9(3):165-9.

[31] Jagdev MK, Gupte S, Aggarwal P, et al. Speciation and drug susceptibility testing including minimum inhibitory concentration for vancomycin in clinical isolates of Enterococci. J Evolution Med Dent Sci 2016; 5(99):7276-9.

[32] Mendiratta DK, Kaur H, Deotale V, et al. Status of high level aminoglycoside resistant Enterococcus faecium and Enterococcus faecalis in a rural hospital of central India. Indian J Med Microbiol 2008; 26(4):369-71. 